\title{
SPECTRAL SETS FOR NUMERICAL RANGE
}

\author{
Hubert Klaja, JAVAd Mashreghi AND ThOMAS RANSFORD
}

Abstract. We define and study a numerical-range analogue of the notion of spectral set. Among the results obtained are a positivity criterion and a dilation theorem, analogous to those already known for spectral sets. An important difference from the classical definition is the role played in the new definition by the base point. We present some examples to illustrate this aspect.

Mathematics subject classification (2010): 47A12, 47A25.

Keywords and phrases: Numerical range, spectral set, dilation.

\section{REFERENCES}

[1] J. Agler, J. Harland AND B. J. RaphaEl, Classical function theory, operator dilation theory, and machine-computation on multiply connected domains, Mem. Amer. Math. Soc. 191 (2008).

[2] C. A. Berger, Normal dilations, Thesis, Cornell University, 1963.

[3] C. A. Berger, A strange dilation theorem, Abstract 625-152, Notices Amer. Math. Soc. 12 (1965), 590.

[4] C. A. Berger, J. G. Stampfli, Mapping theorems for the numerical range, Amer. J. Math. 89 (1967), 1047-1055.

[5] M. CrouzeiX, private communication.

[6] S. W. DRURY, Symbolic calculus of operators with unit numerical radius, Lin. Alg. Appl. 428 (2008), 2061-2069.

[7] C. FoIAş, Certaines applications des ensembles spectraux. I. Mesure harmonique-spectrale, Acad. R. P. Romîne. Stud. Cerc. Mat. 10 (1959), 365-401.

[8] K. E. Gustafson, D. K. M. RaO, Numerical Range. The Field of Values of Linear Operators and Matrices, Springer-Verlag, New York, 1997.

[9] P. R. Halmos, Introduction to Hilbert Space and the Theory of Spectral Multiplicity, reprint of the second (1957) edition, AMS Chelsea Publishing, Providence, RI, 1998.

[10] T. KATO, Some mapping theorems for the numerical range, Proc. Japan Acad. 41 (1965), 652-655.

[11] H. Klaja, J. MAShreghi, T. RANSFORD, On mapping theorems for numerical range, Proc. Amer. Math. Soc. 144 (2016), 3009-3018.

[12] A. LeBow, On von Neumann's theory of spectral sets, J. Math. Anal. Appl. 7 (1963), 64-90.

[13] V. Paulsen, Completely Bounded Maps and Operator Algebras, Cambridge University Press, Cambridge, 2002.

[14] V. Simoncini, D. B. Szyld, On the field of values of oblique projections, Lin. Alg. Appl. 433 (2010), 810-818.

[15] B. Sz.-Nagy, Sur les contractions de l'espace de Hilbert, Acta Sci. Math. Szeged 15 (1953), 87-92.

[16] J. von Neumann, Eine Spektraltheorie für allgemeine Operatoren eines unitären Raumes, Math. Nachr. 4 (1951), 258-281. 